\title{
Irreversibility of Nerve Root Sedimentation Sign in Lumbar Spinal Stenosis After Decompression Surgery: Incidence and Risk Factors
}

Jinhong Zhang ( $\nabla$ drzhangjinhong@sina.com )

Suqian First Hospital

Qunhu Zhang

Suqian first hospital

Shaoxiang Xia

Peking University First Hospital

Yunshan Xu

Southern Medical University Nanfang Hospital

Jinrong Ni

Suqian first hospital

Guoyong Yin

Nanjing Medical University

Xiaojian Cao

Nanjing Medical University

Research article

Keywords: lumbar spinal stenosis, sedimentation, TLIF surgery, cross-sectional area

Posted Date: May 28th, 2020

DOI: https://doi.org/10.21203/rs.3.rs-29922/v1

License: (9) (i) This work is licensed under a Creative Commons Attribution 4.0 International License. Read Full License 


\section{Abstract}

Background. The nerve root sedimentation sign (Sed-sign) is a new diagnostic test for lumbar spinal stenosis. However, few studies have evaluated radiographical outcomes of positive Sed-sign after decompression surgery. The current study aims to evaluate the radiographicaland clinical outcomes of Sed-signafter decompression surgery among a relatively large sample size

Methods. Patients with positive Sed-sign who underwent transforaminal lumbar interbody fusion (TLIF) surgery were reviewed. Based on postoperative MRI scans, patients with negative or irreversible Sed-sign were assigned to the reversible (R) or irreversible (Ir) groups, respectively. Demographics, radiographic parameters, and health-related quality of life (HRQOL) were evaluated.

Results. The R and Ir groups were comprised of 67 and 15 patients, respectively. There were no significant differences between two groups in terms of mean age, sex, postoperative complications as well as mean follow-up time (all $P>0.05$ ). After surgery, disc height $(\mathrm{DH})$, foramina height $(\mathrm{FH})$, and segmental lordosis $(\mathrm{SL})$ in both groups were restored. At the latest follow-up, the smallest cross-sectional area (CSA) was significantly increased when compared with preoperatively $(P<0.05)$. The CSA value and correction rate in the R-group was considerably higher than in the Ir-group $(P=0.002$ and $P=0.007)$. Both groups had improved HRQOL after TLIF surgery; however, the Oswestry disability index, Zürich Claudication Questionnaire, and visual analog scale for back and leg pain were higher in the Ir vs. R groups $(P<0.05)$. Multiple logistic regression analysis revealed that a postoperative CSA less than $131.4 \mathrm{~mm}^{2}$ was a risk factor for the irreversibility of Sed-sign.

Conclusion.TLIF surgery resulted in reversibility of positive Sed-sign for most patients. The irreversibility of Sed-sign was associated with poor clinical outcomes. Independent risk factor for the irreversibility of Sed-sign was CSA less than $131.4 \mathrm{~mm}^{2}$.

\section{Background}

Lumbar spinal stenosis (LSS) has become one of the most common reasons for spinal surgery in the aging population ${ }^{1}$. Success in surgery for these patients had mainly depended on an accurate diagnosis and decompression of central, lateral recess, and foraminal stenosis ${ }^{2,3}$. In past decades, the diagnosis of LSS has depended on radiological and clinical tests such as the cross-sectional area (CSA) of dural sac as well as walking distance ${ }^{4,5}$. Barz et al. ${ }^{6}$ introduced a new morphological sign namely nerve root sedimentation sign (Sed-sign), which was defined as presence of lumbar nerve roots floating in dural sac in at least 1 axial image. With the newly proposed Sed-sign, there has been an attempt to improve the identification of patients with clinically relevant central $\mathrm{LSS}^{6}$.

The incidence of positive Sed-sign was reported from $23-56 \%$ in a previous study ${ }^{6-8}$. By comparing surgical outcomes between patients showing positive and negative Sed-sign, Barz et al. ${ }^{9}$ reported that the Sed-sign did not appear to predict better clinical outcomes. Whereas in non-surgically treated patients, a 
positive sign was found to be associated with more limited improvement ${ }^{9}$. Thus, surgery was proposed for patients with positive Sed-sign. Compared with the influence of preoperative Sed-sign on postoperative surgical outcomes, only a limited number of studies have evaluated whether surgical decompression leads to reversal of the positive Sed-sign. Barz et al. ${ }^{10}$ analyzed 30 patients with preoperative positive Sed-sign who underwent decompression surgery and found that 27 of them had negative Sed-sign at 3 months postoperative MRI evaluation. However, due to the limited sample size in their study, the influence of the positive Sed-sign after surgery on patient-reported outcomes remains unknown. Further, it is essential to quantify the risk factors associated with the irreversible Sed-sign.

Hence, the current study aims to: (1) evaluate the radiographical outcomes of Sed-sign after decompression surgery among a relatively large sample size; (2) compare clinical outcomes between patients with postoperative negative and positive Sed-sign; and (3) further identify risk factors associated with the irreversible positive Sed-sign.

\section{Methods}

\section{Subjects}

This retrospective case series study was approved by our hospital's institutional review board. 223 patients who were identified with LSS at our hospital between January 2011 and December 2017 were identified. Inclusion criteria for this study included: (1) age 50-80 years; (2) central or combined LSS confirmed by radiologic examination on cross-sectional MRI; (3) diagnosis with preoperative Sed-sign; (4) undergoing single-level transforaminal lumbar interbody fusion (TLIF) surgery; and (5) MRI examination at 1-year post-operation. Any patient with one or more of the following criteria was excluded: (1) history of prior lumbar trauma, tumor, infection, and revision surgery; (2) patients with LSS at L5-S1 level; (3) concomitant lumbar scoliosis $>10^{\circ}$; and (4) incomplete demographic data and radiographs.

Each patient's demographic and clinical data, including age, sex, and diagnosis, were obtained using electronic medical records.

\section{Surgical methods}

Surgeries were performed by our single surgeon's team. All patients underwent continuous conservative treatment including medication and physical therapy for a minimum of 6 weeks before the surgery. After standard posterior exposure with subperiosteal dissection of the posterior elements, pedicle screws were placed. Then, adequate decompression was performed through the dominant side of the symptoms. After complete decompression and careful endplate preparation, temporary rod fixations were placed bilaterally with a distraction of the disc space. After that, a polyetheretherketone cage, packed with a mixture of demineralized bone matrix and local bone, was positioned on the anterior rim of the vertebral body. Finally, permanent rods were bilaterally fixed with proper bilateral compression to better restore lordosis. 


\section{Radiographic evaluation}

MRI scans (T2-weighted transverse layers of $4 \mathrm{~mm}$ thickness; Siemens Symphony $1.5 \mathrm{~T}$ ) were performed twice for each patient on 2 different days by 2 experienced independent operators. Using a picture archiving and communications system (PACS) with integrated digital area measurement (GE Healthcare), the smallest CSA of the dural sac was measured on 3 different MRI scans at surgical level by an experienced independent radiologist who was unaware of the Sed-sign as well as other additional clinical information. The mean value of the 3 measurements was calculated. The diagnosis of Sed-sign was based on the MRI scans at the pedicle level. A positive Sed-sign was defined as the absence of nerve root sedimentation in at least 1 transverse MRI scan, at a level above or below, disregarding the location of the scan within the level and its proximity to the maximal stenosis ${ }^{6}$.

In addition to the previously mentioned parameters on MRI scans, the following parameters were measured on X-rays taken preoperatively and at 1-year postoperatively: (1) segmental lordosis (SL), the angle subtended by the superior endplate line of $L 4$ and the inferior endplate line of $L 5$; (2) disc height $(\mathrm{DH})$, the mean value of the anterior and posterior margins of the intervertebral space; and (3) foramina height $(\mathrm{FH})$, the distance between the inferior pedicle wall above the index disc space to the superior pedicle wall from the level below.

In this study, all radiographs were measured by two well-trained senior residents, and the mean of both measurements was adopted for analysis. The interobserver reproducibility of all measurements obtained was tested and quantified by the intraclass correlation coefficient (ICC). There were strong interobserver agreements for all parameters, as all ICCs exceeded 0.8 .

\section{Study group}

Patients with preoperative positive Sed-sign and postoperative negative Sed-sign were assigned to the reversible group (R-group). The remaining patients with postoperative positive Sed-sign were assigned to the irreversible group (Ir-group).

\section{Health-related quality of life (HRQOL)}

HRQOL was assessed using the Oswestry disability index scale (ODI), Zürich Claudication Questionnaire (ZCQ), and visual analog scale (VAS). All questionnaires were completed independently by the patients before surgery and at the latest follow-up.

\section{Statistical analysis}

All data were analyzed using SPSS software (version 20.0; USA). The statistical data are presented as mean \pm standard deviation. The normally distributed data were compared between groups using an unpaired $t$ test or Chi-square tests and within groups using a paired $t$ test. Factors related to the irreversibility of Sed-sign were further investigated using multiple logistic regression analysis. $P$ values < 0.05 were considered statistically significant. 


\section{Results}

A total of 82 patients with mean age of $59.9 \pm 10.2$ years were enrolled. There were 12 patients who underwent TLIF at L2/3, 22 patients at L3/4, and 48 patients at L4/5, respectively. The average follow-up was $39.2 \pm 12.7$ months (range 26-49 months). Based on the MRI scans at the latest follow-up, 67 patients had negative Sed-sign, and were assigned to the R-group. The remaining 15 patients with positive Sed-sign were assigned to the Ir-group. The incidence of irreversibility of positive Sed-sign was $18.2 \%$.

As summarized in Table 1, the characteristics of the R-group and the Ir-group did not differ statistically in terms of mean age, sex, postoperative complications, and follow-up time (all $P>0.05$ ).

Table 1

Patient Data

\begin{tabular}{|c|c|c|c|}
\hline & $\begin{array}{l}\text { R group } \\
(n=67)\end{array}$ & $\begin{array}{l}\text { Ir group } \\
(n=15)\end{array}$ & $P$ value \\
\hline Age, years & $59.1 \pm 12.7$ & $63.9 \pm 13.4$ & 0.193 \\
\hline Sex, female/ male & $28 / 39$ & $8 / 7$ & 0.416 \\
\hline Follow-up, months & $38.8 \pm 11.5$ & $41.5 \pm 14.3$ & 0.434 \\
\hline \multicolumn{4}{|c|}{ Postoperative complications } \\
\hline Incidental durotomy & 3 & 1 & \multirow[t]{4}{*}{0.617} \\
\hline Superficial infection & 4 & 1 & \\
\hline Transient sciatica & 3 & 1 & \\
\hline Screw loosening & 2 & 0 & \\
\hline
\end{tabular}

\section{Radiographical outcomes}

As shown in Table 2, the preoperative smallest CSA was $56.3 \pm 14.1 \mathrm{~mm}^{2}$ in the R-group and $59.2 \pm 13.7$ $\mathrm{mm}^{2}$ in the Ir-group, respectively. There were no significant differences between the two groups in terms of $\mathrm{DH}, \mathrm{FH}$, and $\mathrm{SL}$ (all $\mathrm{P}>0.05$ ). After surgery, $\mathrm{DH}, \mathrm{FH}$, and $\mathrm{SL}$ in both groups were well restored (all $\mathrm{P}<$ 0.05), and remained steady during follow-up. The comparison analyses revealed that no significant difference was observed in terms of $\mathrm{DH}, \mathrm{FH}$, and SL between the R-group and Ir-group (all P>0.05). At the latest follow-up, the smallest CSA was significantly increased when compared with preoperative CSA, with an increase of $60.7 \pm 10.2 \%$ in the R-group and $52.0 \pm 14.3 \%$ in the Ir-group, respectively. The value of the postoperative CSA $(143.5 \pm 23.3$ vs. $123.5 \pm 19.5, P=0.002)$, as well as the correction rate in the $\mathrm{R}$ group $(60.7 \pm 10.2 \%$ vs. $52.0 \pm 14.3, P=0.007)$, was considerably higher than those in the Ir-group. 
Table 2

Comparison of sagittal alignment between $\mathrm{R}$ group and Ir group

\begin{tabular}{|llll|}
\hline & $\begin{array}{l}\text { R group } \\
(\mathbf{n}=\mathbf{6 7})\end{array}$ & $\begin{array}{l}\text { Ir group } \\
(\mathbf{n}=\mathbf{1 5})\end{array}$ & P value \\
\hline CSA, mm ${ }^{2}$ & & & \\
\hline Preoperative & $56.3 \pm 14.1$ & $59.2 \pm 13.7$ & 0.471 \\
\hline Latest follow-up & $143.5 \pm 23.3$ & $123.5 \pm 19.5$ & 0.002 \\
\hline Correction rate (\%) & $60.7 \pm 10.2$ & $52.0 \pm 14.3$ & 0.007 \\
\hline DH, mm & & & 0.600 \\
\hline Preoperative & $9.9 \pm 2.7$ & $9.5 \pm 2.5$ & 0.819 \\
\hline Postoperative & $12.5 \pm 3.0$ & $12.7 \pm 3.3$ & 0.801 \\
\hline Latest follow-up & $12.1 \pm 2.6$ & $12.3 \pm 3.5$ & 0.492 \\
\hline FH, mm & & & 0.752 \\
\hline Preoperative & $17.4 \pm 3.4$ & $18.1 \pm 4.2$ & 0.575 \\
\hline Postoperative & $19.9 \pm 4.5$ & $20.3 \pm 4.0$ & 0.483 \\
\hline Latest follow-up & $19.5 \pm 3.7$ & $20.1 \pm 3.9$ & 0.365 \\
\hline SL, ${ }^{\circ}$ & & & $13.2 \pm 2.3$ \\
\hline Preoperative & $10.2 \pm 3.6$ & $11.1 \pm 2.7$ & \\
\hline Postoperative & $12.9 \pm 2.7$ & & \\
\hline Latest follow-up & $12.6 \pm 3.1$ & & \\
\hline CSA, cross-sectional area; $\mathrm{DH}$, disc height; FH, foramina height; SL, segmental lordosis & & \\
\hline OL OUtCOMeS & & & \\
\hline
\end{tabular}

The distribution of scores for HRQOL is shown in Table 3. There were no significant differences between the two groups in terms of preoperative ODI, ZCQ physical function and symptom severity domain, VASback, and leg pain. At the latest follow-up, both groups improved notably. However, the ODI, ZCQ physical function and symptom severity domain, VAS-back, and leg pain in the Ir-group were significantly higher than those in the R-group $(P<0.05)$. 
Table 3

Comparison of HRQOL between R group and Ir group

\begin{tabular}{|c|c|c|c|}
\hline & $\begin{array}{l}\text { R group } \\
(n=67)\end{array}$ & $\begin{array}{l}\text { Ir group } \\
(n=15)\end{array}$ & $P$ value \\
\hline \multicolumn{4}{|l|}{ ODI } \\
\hline Preoperative & $42.2 \pm 6.9$ & $40.1 \pm 7.3$ & 0.294 \\
\hline Latest follow-up & $15.3 \pm 4.9$ & $18.9 \pm 5.5$ & 0.013 \\
\hline \multicolumn{4}{|c|}{ ZCQ-physical function domain } \\
\hline Preoperative & $2.7 \pm 1.2$ & $2.6 \pm 1.0$ & 0.765 \\
\hline Latest follow-up & $1.4 \pm 0.4$ & $1.8 \pm 0.7$ & 0.003 \\
\hline \multicolumn{4}{|c|}{ ZCQ-symptom severity domain } \\
\hline Preoperative & $3.3 \pm 1.1$ & $3.5 \pm 0.9$ & 0.513 \\
\hline Latest follow-up & $1.8 \pm 0.5$ & $2.3 \pm 0.6$ & 0.001 \\
\hline \multicolumn{4}{|l|}{ VAS-BP } \\
\hline Preoperative & $6.6 \pm 1.5$ & $7.1 \pm 1.8$ & 0.264 \\
\hline Latest follow-up & $1.3 \pm 0.9$ & $2.0 \pm 0.8$ & 0.006 \\
\hline \multicolumn{4}{|l|}{ VAS-LP } \\
\hline Preoperative & $6.7 \pm 1.7$ & $6.2 \pm 1.4$ & 0.292 \\
\hline Latest follow-up & $1.6 \pm 0.8$ & $2.2 \pm 0.9$ & 0.012 \\
\hline
\end{tabular}

\section{Regression Analysis}

Unpaired $t$ test and Chi-square test results showed that probable risk factors for the irreversibility of Sedsign were the surgical method, postoperative CSA, and the correction rate of CSA. Logistic regression analyses of the three probable risk factors revealed that the risk factor for the irreversibility of Sed-sign was the postoperative CSA, with the cut-off value at $131.4 \mathrm{~mm}^{2}(\mathrm{OR}, 0.561 ; 95 \%$ confidence interval, $0.113-0.871 ; P=0.003)$.

\section{Discussion}

Since Sed-sign was first introduced by Barz et $\mathrm{al}^{6}$, an important research focus has been on the evaluation of clinical and radiological characteristics to better distinguish LSS from lower back pain patients. Due to the relatively lower sensitivity in detecting clinically defined LSS in orthopedic clinical 
cohorts, the Sed-sign does not allow for definitive differential diagnosis of LSS $^{11,12}$. Compared with nonsurgically treated positive Sed-sign patients, favorable outcomes have been reported for patients who underwent surgical treatment ${ }^{9,13}$. Therefore, Sed-sign seemed to be an indication to help determine the appropriate treatment. Previous studies investigating the Sed-sign have concentrated on the diagnosis of LSS as well as its influence on the surgical outcomes. Evaluation of the reversibility of the Sed-sign after decompression surgery as well as the influence of irreversibility on surgical outcomes is still mandatory.

The current study retrospectively reviewed 82 patients with LSS who underwent surgical decompression at our spine center. The incidence of positive Sed-sign in the current study was similar to that reported in the literature ${ }^{6-8}$. The definitive disease mechanism for positive Sed-sign remains unknown. The increased epidural pressure at the level of spinal stenosis has been reported as the main cause for positive Sed-sign in patients with $\mathrm{LSS}^{14}$. Barz et al. ${ }^{14}$ noted that increased epidural pressure at the level of the stenosis may result in tethering of nerve roots in the spinal canal at that level as if held by a mechanical clamp. In the current study, $80.4 \%$ of patients showed reversibility of the Sed-sign after decompression surgery performed at the level of stenosis. This tendency was similar to the study performed by Barz et al. ${ }^{10}$, who reported that 27 of 30 patients had negative Sed-sign after decompression surgery. Together, these results suggested that decompression surgery resulted in reversibility of Sed-sign in most patients. However, it should be noted that not all LSS patients with positive Sed-sign showed reversibility after decompression surgery (Fig. 1). The frequency of patients with positive Sed-sign after decompression surgery in the current study was slightly higher than the study of Barz et al. ${ }^{10}$ This difference may be due to the fact that only patients who underwent single-level decompression surgery were enrolled in the current study.

Barz et al. ${ }^{9}$ reported that a positive Sed-sign was associated with limited clinical improvement in nonsurgically treated patients. Among patients treated with decompression surgery, those with positive Sedsign achieved similar clinical improvements when compared with negative Sed-sign patients ${ }^{9}$. HRQOL in the current study, based on the ODI, VAS, and ZCQ scores, suggested that both R and Ir groups achieved improvements after surgery compared with the preoperative evaluations. This finding was consistent with those of Barz et al. ${ }^{10}$, who reported that patients with the irreversibility of Sed-sign made improvements in clinical outcomes for ODI, VAS, and ZCQ scores. In the current study, a further comparison between patients with positive and negative Sed-sign after decompression surgery showed that the ODI, VAS, and ZCQ scores in the Ir group were significantly lower than those in the R group. Our findings suggested that the irreversibility of Sed-sign after decompression surgery was associated with poorer clinical outcomes when compared with those with negative Sed-sign at post-operation.

The main mechanisms underlying the irreversibility of positive Sed-sign remain unknown. Possible reasons for positive Sed-sign included insufficient decompression, postoperative hematoma, excessive postoperative scar tissue, and epidural lipomatosis ${ }^{10}$. Barz et al. ${ }^{10}$ noted that the reasons for 3 patients with irreversibility of Sed-sign were potentially associated with postoperative complications, including two cases with epidural fat and one case with an epidural cyst. In fact, such complications may also be 
attributed to abnormal epidural pressure, like insufficient decompression of the LSS. In the current study, similar complication rates were found between those with reversibility and irreversibility of Sed-sign. Based on the multiple logistic regression, our results showed that the primary risk factor for the irreversibility of positive Sed-sign was a postoperative CSA of less than $131.4 \mathrm{~mm}^{2}$. The association between smaller CSA and the irreversibility of Sed-sign may also attribute to the increased epidural pressure, which may be induced by the relatively smaller CSA. Therefore, our results further demonstrated the relationship between increased epidural pressure and formation of positive Sed-sign.

The clinical relevance of this study relates to decreasing the incidence of the irreversibility of Sed-sign and improving HRQOL after decompression surgery. First, decompression at the stenosis level should be performed. Second, postoperative complications, such as epidural fat and hematoma, may also increase abnormal epidural pressure and further influence the reversibility of preoperative Sed-sign. Therefore, complications should be identified and managed as soon as possible.

There were also some limitations in the current study. First, the sample size in the current study was relatively small. Patients enrolled in the current study only underwent single-level TLIF decompression for consistency. The relationship between multi-level decompression and reversibility of positive Sed-sign remains unclear. Second, HRQOL may be influenced by several sagittal parameters. However, further evaluation of sagittal alignments, such as pelvic incidence-lumbar lordosis, was limited due to the retrospective character of the current study. Third, even all of these surgeries were performed by same surgical team and the goal of surgery is to achieve adequate decompression, the effect of insufficient decompression on HRQOL should not be neglected. Therefore, a larger sample size with a detailed evaluation of sagittal alignment should be performed in future studies.

\section{Conclusions}

Decompression surgery resulted in reversibility of positive Sed-sign for most patients. HRQOL in patients with negative Sed-sign was notably better than those with the irreversibility of Sed-sign. An independent risk factor for the irreversibility of Sed-sign was a CSA less than $131.4 \mathrm{~mm}^{2}$. This information should be taken into consideration during surgical decision making.

\section{Abbreviations}

Sed-sign

sedimentation sign; TLIF:transforaminal lumbar interbody fusion; HRQOL:health-related quality of life; CSA:cross-sectional area; LSS:Lumbar spinal stenosis; SL:segmental lordosis; DH:disc height; FH:foramina height; ICC:intraclass correlation coefficient; ODI:Oswestry disability index scale; VASLP:visual analog scale for leg pain; VAS-BP:visual analog scale for back pain

\section{Declarations}




\section{Acknowledgements}

No

\section{Funding}

No

\section{Author's contributions}

Jinhong Zhang, MD, Writing the paper, Data collection, Performed surgeries, Study design,

Qunhu Zhang, MD, Performed surgeries, Study design.

Shaoxiang Xia, MD, Data collection, Data analysis

Yunshan Xu, MD, Performed surgeries, Data collection, Data analysis

Jinrong Ni, MD, Data collection, Data analysis

Guoyong Yin, Performed surgeries, Study design,

Xiaojian Cao, MD, Performed surgeries, Study design,

\section{Availability of data and materials}

All data generated or analyzed during this study are presented in themanuscript. Please contact the corresponding author for access to datapresented in this study.

\section{Ethics approval and consent to participate}

The present study was approved by the local ethics committee of Suqian First Hospital

\section{Consent for publication}

Agree

\section{Competing interests}

No other conflicts of interest, financial orotherwise, are declared by the author(s). 


\section{References}

1. Kim HJ, Lee JI, Kang KT, et al. Influence of pain sensitivity on surgical outcomes after lumbar spine surgery in patients with lumbar spinal stenosis. Spine. 2015;40:193-200.

2. Kim YK, Lee JW, Kim HJ, et al. Diagnostic advancement of axial loaded lumbar spine MRI in patients with clinically suspected central spinal canal stenosis. Spine. 2013;38:E1342-7.

3. Sigmundsson FG, Jonsson B, Stromqvist B. Preoperative pain pattern predicts surgical outcome more than type of surgery in patients with central spinal stenosis without concomitant spondylolisthesis: a register study of 9051 patients. Spine. 2014;39:E199-210.

4. Kitab S, Lee BS, Benzel EC. Redefining lumbar spinal stenosis as a developmental syndrome: an MRIbased multivariate analysis of findings in 709 patients throughout the 16- to 82-year age spectrum. Journal of neurosurgery. Spine 2018:1-7.

5. Wang X, Wang H, Sun $\mathrm{C}$, et al. Analysis of radiological parameters associated with decreased fractional anisotropy values on diffusion tensor imaging in patients with lumbar spinal stenosis. European spine journal: official publication of the European Spine Society, the European Spinal Deformity Society, and the European Section of the Cervical Spine Research Society 2018.

6. Barz T, Melloh M, Staub LP, et al. Nerve root sedimentation sign: evaluation of a new radiological sign in lumbar spinal stenosis. Spine. 2010;35:892-7.

7. Laudato PA, Kulik G, Schizas C. Relationship between sedimentation sign and morphological grade in symptomatic lumbar spinal stenosis. European spine journal: official publication of the European Spine Society the European Spinal Deformity Society the European Section of the Cervical Spine Research Society. 2015;24:2264-8.

8. Tomkins-Lane CC, Quint DJ, Gabriel S, et al. Nerve root sedimentation sign for the diagnosis of lumbar spinal stenosis: reliability, sensitivity, and specificity. Spine. 2013;38:E1554-60.

9. Barz T, Staub LP, Melloh M, et al. Clinical validity of the nerve root sedimentation sign in patients with suspected lumbar spinal stenosis. The spine journal: official journal of the North American Spine Society. 2014;14:667-74.

10. Barz C, Melloh M, Staub LP, et al. Reversibility of nerve root sedimentation sign in lumbar spinal stenosis patients after decompression surgery. European spine journal: official publication of the European Spine Society the European Spinal Deformity Society the European Section of the Cervical Spine Research Society. 2017;26:2573-80.

11. Fazal A, Yoo A, Bendo JA. Does the presence of the nerve root sedimentation sign on MRI correlate with the operative level in patients undergoing posterior lumbar decompression for lumbar stenosis? The spine journal: official journal of the North American Spine Society. 2013;13:837-42.

12. Zhang $L$, Chen R, Liu B, et al. The nerve root sedimentation sign for differential diagnosis of lumbar spinal stenosis: a retrospective, consecutive cohort study. European spine journal: official publication of the European Spine Society the European Spinal Deformity Society the European Section of the Cervical Spine Research Society. 2017;26:2512-9. 
13. Moses RA, Zhao W, Staub LP, et al. Is the sedimentation sign associated with spinal stenosis surgical treatment effect in SPORT? Spine 2015;40:129 - 36.

14. Barz T, Melloh M, Staub LP, et al. Increased intraoperative epidural pressure in lumbar spinal stenosis patients with a positive nerve root sedimentation sign. European spine journal: official publication of the European Spine Society the European Spinal Deformity Society the European Section of the Cervical Spine Research Society. 2014;23:985-90.

\section{Figures}
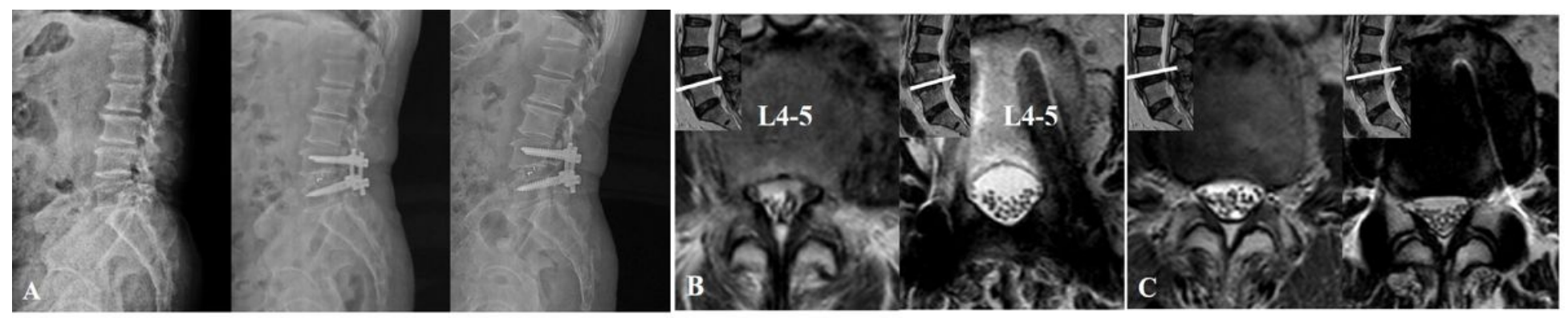

\section{Figure 1}

A Standing radiographs from a 62-year-old female with lumbar stenosis who received surgical decompression with transforaminal lumbar interbody fusion. B After surgery, the stenosis was well restored. C However, the positive sedmentation sign were still diagnosed at the latest follow-up. 